Research Article

\title{
Incorporating the Number of PLN into the AJCC Stage Could Better Predict the Survival for Patients with NSCLC: A Large Population-Based Study
}

\author{
Xiaoling Shang $\mathbb{D}^{1,2}$ Zhenxiang Li ${ }^{1},{ }^{3}$ Jiamao Lin $\mathbb{D}^{\circ},{ }^{4}$ Haining Yu $\mathbb{D}^{5},{ }^{5}$ Chenglong Zhao $\mathbb{D}^{6}$, \\ Haiyong Wang $\mathbb{D}^{4}$ and Jian Sun $\mathbb{B D}^{7}$ \\ ${ }^{1}$ Shandong Cancer Hospital and Institute, Cheeloo College of Medicine, Shandong University, Jinan, Shandong 250012, China \\ ${ }^{2}$ Department of Clinical Laboratory, Shandong Cancer Hospital and Institute, \\ Shandong First Medical University and Shandong Academy of Medical Sciences, Shandong University, Jinan 250117, China \\ ${ }^{3}$ Department of Radiation Oncology, Shandong Cancer Hospital and Institute, \\ Shandong First Medical University and Shandong Academy of Medical Sciences, Jinan 250117, China \\ ${ }^{4}$ Department of Internal Medicine-Oncology, Shandong Cancer Hospital and Institute, \\ Shandong First Medical University and Shandong Academy of Medical Sciences, Jinan 250117, China \\ ${ }^{5}$ Personnel Division, Shandong Cancer Hospital and Institute, \\ Shandong First Medical University and Shandong Academy of Medical Sciences, Jinan 250117, China \\ ${ }^{6}$ Department of Pathology, Shandong Cancer Hospital and Institute, \\ Shandong First Medical University and Shandong Academy of Medical Sciences, Jinan 250117, China \\ ${ }^{7}$ Department of Thoracic Surgery, Shandong Cancer Hospital and Institute, \\ Shandong First Medical University and Shandong Academy of Medical Sciences, Jinan 250117, China
}

Correspondence should be addressed to Haiyong Wang; wanghaiyong6688@126.com and Jian Sun; drsunjian0168@sina.com

Received 13 April 2020; Revised 23 October 2020; Accepted 9 November 2020; Published 11 December 2020

Academic Editor: Pierfrancesco Franco

Copyright ( 92020 Xiaoling Shang et al. This is an open access article distributed under the Creative Commons Attribution License, which permits unrestricted use, distribution, and reproduction in any medium, provided the original work is properly cited.

Purpose. This study aimed to investigate the application of the number of positive lymph nodes (PLNs) in tumor, node, metastasis (TNM) staging system of non-small cell lung cancer (NSCLC) patients. Patients and Methods. We screened a total of 15820 patients with resected NSCLC between 2004 and 2015 from SEER database. The X-tile model was used to determine the cutoff values of the number of PLNs. Overall survival (OS) curves were plotted using the Kaplan-Meier method, and the differences among the individual groups were defined using the log-rank test. Cox regression model was used to perform univariate and multivariate analyses and to assess the association between the number of PLNs and OS. Results. In this study, using the X-tile model, we screened three different cutoff values, including nN0, nN1-3, and nN4-. Survival curves demonstrated that our defined $\mathrm{nN}$ stage had a significant predictive value for OS $(P<0.001)$. In the univariate and multivariate Cox analyses, the result showed that $\mathrm{nN}$ stage was a significant prognostic factor of OS for NSCLC patients $(P<0.001)$. Subsequently, we classified the patients into five subgroups based on the combination of $\mathrm{pN}$ and $\mathrm{nN}$ stages, including $\mathrm{pN} 0+\mathrm{nN} 0, \mathrm{pN} 1+\mathrm{nN} 1-3, \mathrm{pN} 2+\mathrm{nN} 1-3, \mathrm{pN} 1+\mathrm{nN} 4-$, and $\mathrm{pN} 2+\mathrm{nN} 4-$. Moreover, survival curves revealed significant differences among these five groups $(P<0.001)$. Conclusion. A combination of pathological LNs $(\mathrm{pN})$ and the number of $\mathrm{LN}(\mathrm{nN})$ involvement in NSCLC patients had a better prognostic value than the current TNM staging system based on only $\mathrm{pN}$ stage.

\section{Introduction}

Lung cancer is considered a severe disease worldwide and is the leading cause of cancer death, where $85 \%$ comprises non-small cell lung cancer (NSCLC) [1,2]. According to the 2018 Annual Report of Chinese National Cancer Centre, lung cancer had the highest incidence and mortality rates among all cancers. Thus, accurate staging, proper treatment, 
and better prognosis are essential to improve the survival of NSCLC patients [3].

The tumor, node, metastasis (TNM) staging system, based on primary tumor character (T), nodal involvement $(\mathrm{N})$, and distant metastasis $(\mathrm{M})$, has a significant impact on therapeutic options and prognosis, which is essential in determining ways to deliver care to patients. There were some updates of $\mathrm{T}$ and $\mathrm{M}$ classification in the eighth version, but the $\mathrm{N}$ classification remained unchanged $[4,5]$. Since pathologically positive lymph node (PLN) indicates a higher recurrence risk, accurate assessment of $\mathrm{LN}$ is essential in creating treatment strategy and prognostic care. Even lymphadenectomy is recommended to be performed in lung resection surgery to ensure a significant survival, but still, $25 \%$ to $50 \%$ of early-stage lung cancer patients have a disease recurrence, suggesting that the current staging system is still inaccurately sufficient to assess NSCLC.

PLN was also considered a strong prognostic factor in NSCLC. It has been proven that the number of PLNs has a fundamental prognostic value in gastric, colorectal, breast, bladder, and esophageal cancer [6]. Furthermore, the number of PLNs was recommended as a staging parameter in these cancers by the National Comprehensive Cancer Network guidelines, but it was not systematically illustrated in NSCLC. Only LN stations were elaborated in the latest 8th edition of the TNM staging system, with no more details about the exact number in the classification.

Hence, we used the Surveillance, Epidemiology, and End Results (SEER) database, selected 15,820 NSCLC patients who underwent complete systematic resection of the LNs, and retrospectively investigated the survival rate affected by the PLN number. The X-tile model was used to determine the threshold of LN number. Comprehensively, we attempted to combine the PLN number and the current TNM staging system to provide a more precise treatment guideline and establish a better prognosis.

\section{Patients and Methods}

2.1. Data Source. The SEER database provided a comprehensive source of cancer statistics, including detailed information on patient demographics, pathologic diagnosis, treatment strategy, and prognosis. Using this database, we selected and analyzed a total of 15,820 patients between 2004 and 2015 using the SEER * Stat 8.3.5 software. All the patients had NSCLC without distant metastasis and underwent lung resection surgery involving the dissection of hilar and mediastinal LNs. The number of harvested LNs was at least 10, which would ensure an optimal prognosis and a more accurate staging. Staging was classified according to the 6th edition of the TNM staging system. The patients with incomplete information were all excluded.

2.2. Ethical Statement. This study was performed according to the Declaration of Helsinki. Permission was granted in accessing the SEER database. The Ethics Committee of Shandong Cancer Hospital and Institute approved this study. Personal information was not included. All the data were obtained from the public database which did not involve personal information, so the informed consent was not required.

2.3. Statistical Analyses. The X-tile model was used to determine the cutoff values of the number of PLNs. Survival curves were plotted using the Kaplan-Meier method, and differences among the individual groups were defined using the log-rank test. Cox regression model was used to perform univariate and multivariate analyses and to assess the association between the number of PLNs and OS. All statistical methods were two-sided, and $P<0.05$ was considered to be statistically significant. Statistical Package for the Social Sciences 22.0 software was used in data analysis.

\section{Results}

3.1. Patients' Characteristics. A total of 15,820 NSCLC patients between 2004 and 2015 were selected from the SEER database for analysis as listed in Table 1. Among these, $38.9 \%$ were younger than 65 years old, and $61.1 \%$ were older than 65 years old. Regarding race, $85.2 \%$ were White, $7.8 \%$ were Black, and $6.9 \%$ were from other races. Female patients had a percentage of $47.5 \%$, while male patients had 52.5\%. Additionally, a major group of patients belonged to adenocarcinoma $(60.6 \%)$ and the others to squamous $(39.4 \%)$. The percentages of patients in $\mathrm{T} 1, \mathrm{~T} 2$, $\mathrm{T} 3$, and $\mathrm{T} 4$ stages were $35.2 \%, 50 \%, 6.6 \%$, and $8.2 \%$, respectively. A majority of patients were in stage pN0 $(63.5 \%)$, and the percentage of patients in stages $\mathrm{pN} 1$, $\mathrm{pN} 2$, and $\mathrm{pN} 3$ were $20.4 \%, 15.8 \%$, and $0.3 \%$, respectively. A total of $65.1 \%$ of patients belonged to the nN0 stage, and $23.2 \%$ and $11.7 \%$ patients were in stage $\mathrm{nN1}-3$ and stage $\mathrm{nN} 4$, respectively.

3.2. Determination of Cutoff Values for PLNs. The cutoff values of PLNs were determined using the X-tile model. Survival curves were analyzed using the Kaplan-Meier method, and log-rank test was used to compare the differences. According to LN numbers, the groups were divided into low $(n=0)$, medium $(1 \leq n \leq 3)$, and high $(3<n \leq 61)$ using the X-tile model (Figures 1(a) and 1(b)). Therefore, based on the threshold, three stages were determined as nN0, nN1-3, and nN4-.

3.3. Survival Analysis. By evaluating the influence of PLN number on NSCLC patients, we first analyzed the OS according to the $\mathrm{pN}$ stage. The $\mathrm{pN}$ stage was widely accepted as a prognostic factor, and our data showed a significant difference among the $\mathrm{pN}$ groups $(P<0.001)$ (Figure $2(\mathrm{a})$ ). Using the same method, we found that the OS curve was well distinguished by the defined $\mathrm{nN}$ stage $(P<0.001)$ (Figure 2(b)). These data suggested that our defined $\mathrm{nN}$ stage had a significant predictive value for prognosis. Subsequently, patients with different pathological tumor ( $\mathrm{pT}$ ) classifications were grouped according to $\mathrm{nN}$ status. We plotted survival curves of $\mathrm{nN}$ status based on different $\mathrm{pT}$ stages, including 
TABLE 1: Non-small cell lung cancer (NSCLC) patient characteristics from SEER database.

\begin{tabular}{|c|c|c|}
\hline Variables & Number & $\%$ \\
\hline \multicolumn{3}{|l|}{ Age } \\
\hline$<65$ & 6158 & 38.9 \\
\hline$\geq 65$ & 9662 & 61.1 \\
\hline \multicolumn{3}{|l|}{ Race } \\
\hline White & 13483 & 85.2 \\
\hline Black & 1244 & 7.8 \\
\hline Others & 1093 & 6.9 \\
\hline \multicolumn{3}{|l|}{ Sex } \\
\hline Female & 7513 & 47.5 \\
\hline Male & 8307 & 52.5 \\
\hline \multicolumn{3}{|l|}{ Histology } \\
\hline Adenocarcinoma & 9587 & 60.6 \\
\hline Squamous & 6233 & 39.4 \\
\hline \multicolumn{3}{|l|}{ T stage } \\
\hline $\mathrm{T} 1$ & 5563 & 35.2 \\
\hline $\mathrm{T} 2$ & 7910 & 50.0 \\
\hline $\mathrm{T} 3$ & 1047 & 6.6 \\
\hline $\mathrm{T} 4$ & 1300 & 8.2 \\
\hline \multicolumn{3}{|l|}{$\mathrm{pN}$ stage } \\
\hline $\mathrm{pNO}$ & 10039 & 63.5 \\
\hline pN1 & 3227 & 20.4 \\
\hline $\mathrm{pN} 2$ & 2505 & 15.8 \\
\hline $\mathrm{pN} 3$ & 49 & 0.3 \\
\hline \multicolumn{3}{|l|}{$\mathrm{nN}$ stage } \\
\hline nNo & 10304 & 65.1 \\
\hline $\mathrm{nN} 1-3$ & 3669 & 23.2 \\
\hline nN4- & 1847 & 11.7 \\
\hline
\end{tabular}

pT1, pT2, pT3, and pT4. In Figure 3(a), we found that the survival curves of stages pT1nN0, pT1nN1-3, and pT1nN4were obviously separated and significantly distinguished $(P<0.001)$. Similar results of the survival curves of stages $\mathrm{nN} 0, \mathrm{nN} 1$, and $\mathrm{nN} 4-$ were observed in the pT2, pT3, and pT4 groups (all $P<0.001$ ) (Figures $3(\mathrm{~b})-3(\mathrm{~d})$ ).

3.4. Survival on the $p N$ and the $n N$ Stages. In Table 2, Cox proportional hazards model was used to evaluate the prognostic value of the baseline characteristics. Univariate and multivariate analysis revealed that variables including age, race, sex, histology, $\mathrm{pT}$ stage, and $\mathrm{pN}$ stage were all significant prognostic factors on OS for NSCLC patients (all $P<0.001)$. In multivariate analysis, the $\mathrm{pN}$ stage was an independent prognostic factor for survival ( $\mathrm{pN} 1$ vs. $\mathrm{pN} 0$ : hazard ratio $(\mathrm{HR}), 1.630 ; 95 \%$ confidence interval $(\mathrm{CI})$, 1.534-1.733; $P<0.001$; pN2 vs. pN0: HR, 2.157; 95\% CI, 2.023-2.300; $P<0.001$; and $\mathrm{pN} 3$ vs. $\mathrm{pN} 0: \mathrm{HR}, 2.799 ; 95 \% \mathrm{CI}$, 1.983-3.949; $P<0.001)$.

To further clarify the significance of $\mathrm{nN}$ stage, we then compared different factors including $\mathrm{nN}$ stage on $\mathrm{OS}$ in NSCLC patients. Univariate and multivariate analysis revealed that all the factors, including age, race, sex, histology, pT stage, and $\mathrm{nN}$ stage, were considered to have independent prognostic values (all, $P<0.001$ ). In multivariate analysis, the $\mathrm{nN}$ stage was an independent prognostic factor of OS (nN1-3 stage vs. nN0 stage: $\mathrm{HR}, 1.657$; 95\% CI, 1.564-1.756; $P<0.001$ and nN4- stage vs. nN0 stage: $H R$,
2.371; 95\% CI, 2.213-2.540; $P<0.001)$. Factors that affected the OS using univariate and multivariate analyses are listed in Table 3.

3.5. Survival Curves Based on the Combination of $p N$ and $n N$ Stages. Nodal status is an important factor for TNM staging system. The $\mathrm{pN}$ stage has already exhibited an essential prognostic value and was involved in the classification, but little is known about the value of $\mathrm{nN}$ as a complementary for classification. We classified the patients into five subgroups based on the combination of the $\mathrm{pN}$ and $\mathrm{nN}$ stages, namely, $\mathrm{pN} 0+\mathrm{nN} 0, \mathrm{pN} 1+\mathrm{nN} 1-3, \mathrm{pN} 2+\mathrm{nN} 1-3, \mathrm{pN} 1+\mathrm{nN} 4-$, and $\mathrm{pN} 2+\mathrm{nN} 4-$. Subsequently, we drew survival curves of these subgroups. The result revealed that NSCLC patients among these five groups had significantly different OS (log-rank test, $P<0.001$ ) (Figure 4 ).

\section{Discussion}

The lung cancer TNM staging system is developed based on sophisticated statistical analysis of patients. It defines the anatomical extent of lung cancer, provides the criteria to distinguish specific patients, and makes the clinical cohort studies easier. Stage groups are defined by the specific primary tumor $(\mathrm{T})$, nodal status for metastasis $(\mathrm{N})$, and metastasis at the distant organs (M).

Nodal involvement is a critical factor that could predict the prognosis after surgery $[7,8]$, but there is still no accurate evidence to illustrate the influence of the extent of LN involvement in NSCLC. Although the pathological lymph nodes stage has long been a basic criterion of TNM staging system, it could not evaluate the prognosis of patients more accurately. Particularly, for mediastinal LN involvement, the prognostic values were not well stated. Thus, the border of $\mathrm{N}$ classification was difficult to define due to its ambiguity and complexity. And the TNM staging system needs to be better evaluated and justified.

The number of PLNs has been proven to have significant influence on certain cancers such as gastric, breast, colorectal, and bladder cancer [9-11]. Moreover, $\mathrm{nN}$ had been involved in the classification of these cancers. Studies also showed that $\mathrm{nN}$ was an essential prognostic factor for resected NSCLC [12-15]. Herr et al. [12] showed that $\mathrm{nN}$ staging was a better prognostic indicator than $\mathrm{pN}$ staging. Lee et al. [16] also demonstrated that the number of PLNs was an important prognostic factor for resected NSCLC, consistent with the result of study by Fukui et al. [17]. Similarly, a previous study led by David et al. also demonstrated that the number of LNs sampled (NLNS) influenced both OS and cancer-specific survival (CSS) for NSCLC patients [18]. And the authors concluded that NLNS was a predictor of OS and CSS for NSCLC. All of the above studies have demonstrated that the number of lymph nodes could be used as a predictive and prognostic indicator among NSCLC patients. The $\mathrm{N}$ stage classification in the latest $8^{\mathrm{th}}$ edition had similar content as the previous $7^{\text {th }}$ edition, with only the addition of the subgroup of pathology position, which defined N1 to N1a and N1b, N2 to N2a1, N2a2, and N2b by 


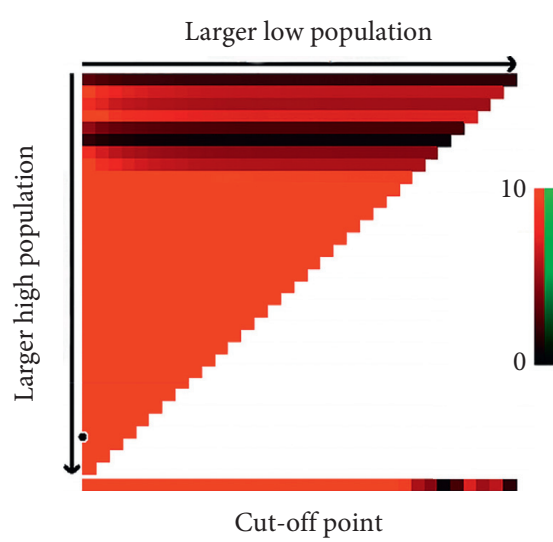

(a)

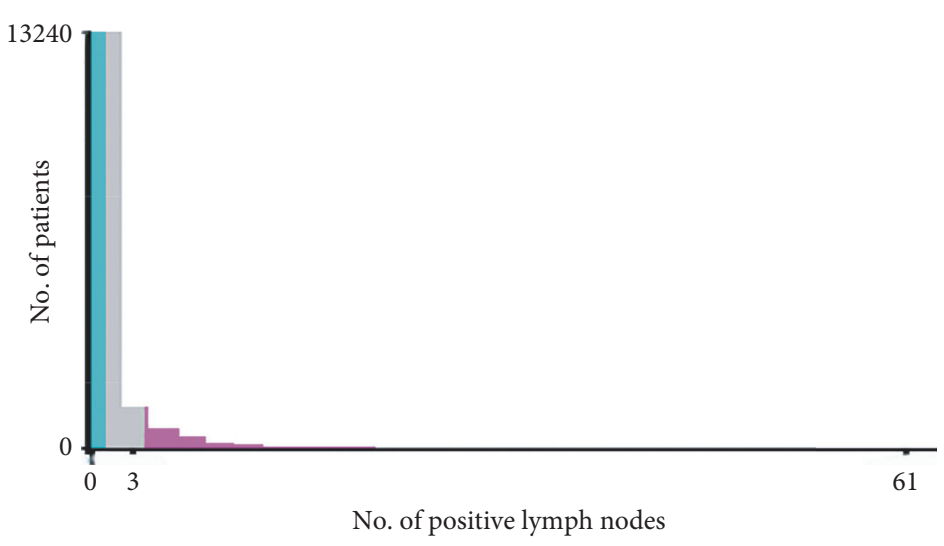

(b)

Figure 1: The optimal threshold of the number of positive lymph nodes determined by the X-tile model. (a) X-tile plots based on no. of positive lymph nodes. (b) The optimal cutoff point is shown by the blue (no. of PLN $=0)$, gray ( $1 \leq$ no. of PLN $\leq 3$ ), and violet panel (no. of PLN $\geq 4$ ).

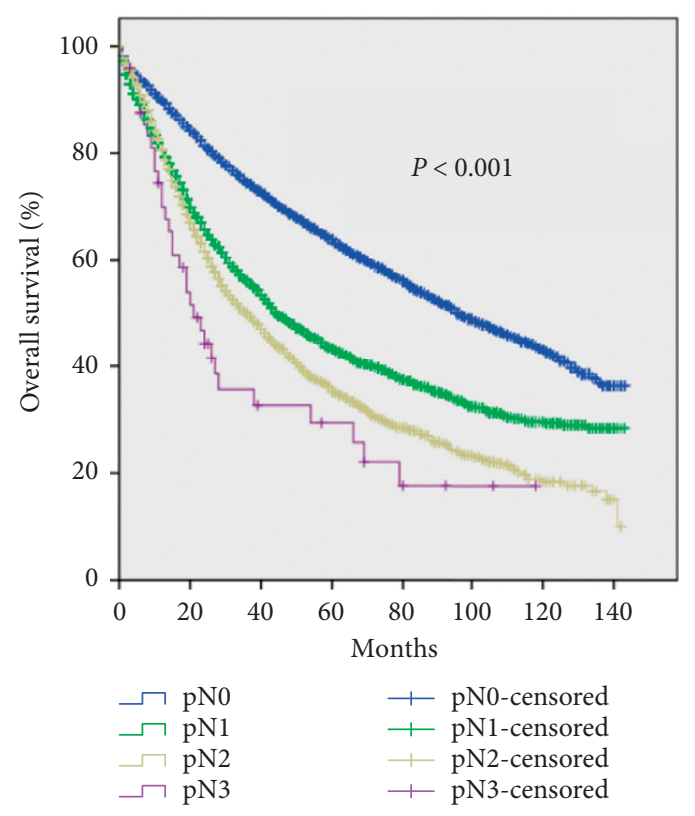

(a)

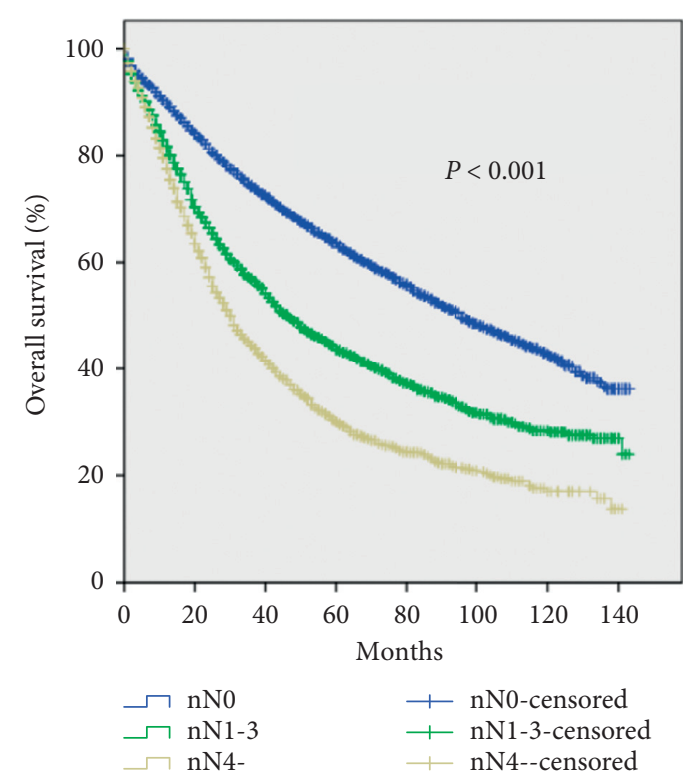

(b)

FIgURE 2: Survival curves of NSCLC patients based on the (a) current $\mathrm{pN}$ stage $(P<0.001)$ and $(\mathrm{b})$ hypothesized $\mathrm{nN}$ stage $(P<0.001)$.

stops of LN involved. However, whether the number of PLNs might be a more accurate disease indicator in NSCLC had not been illustrated. To date, determining the best prognostic index of lung cancer for $\mathrm{nN}$ stage or $\mathrm{pN}$ stage is not yet clear.

According to the staging manual of the Thoracic Oncology of the International Association for the Study of Lung Cancer, at least six LNs/stations should be histologically confirmed to be nonmetastatic and subsequently can be defined as pN0, but it did not mention the exact number of resected LN to predict the prognosis of NSCLC [3]. Saji et al. suggested ten as a cutoff value [14]. Other studies $[19,20]$ showed that 16 examined LNs could better evaluate the disease staging and postoperative care. According to the previous studies, dissected LN number ranges from 10 to 18 . Regarding the calculation of the number of metastatic LNs, optimal surgery should initially be performed. It had been confirmed that there was no significant difference in survival between selective LN dissection and complete LN dissection, but controversy still exists as regards this finding.

Patients had shorter operative time, lesser blood loss, and fewer morbidity rates in selective LN dissection. Therefore, fewer LN resection was recommended to reduce operative risks and to achieve a better postoperative recovery. In this case, we selected patients with at least 10 resected LNs as a criterion in the study. Another critical 


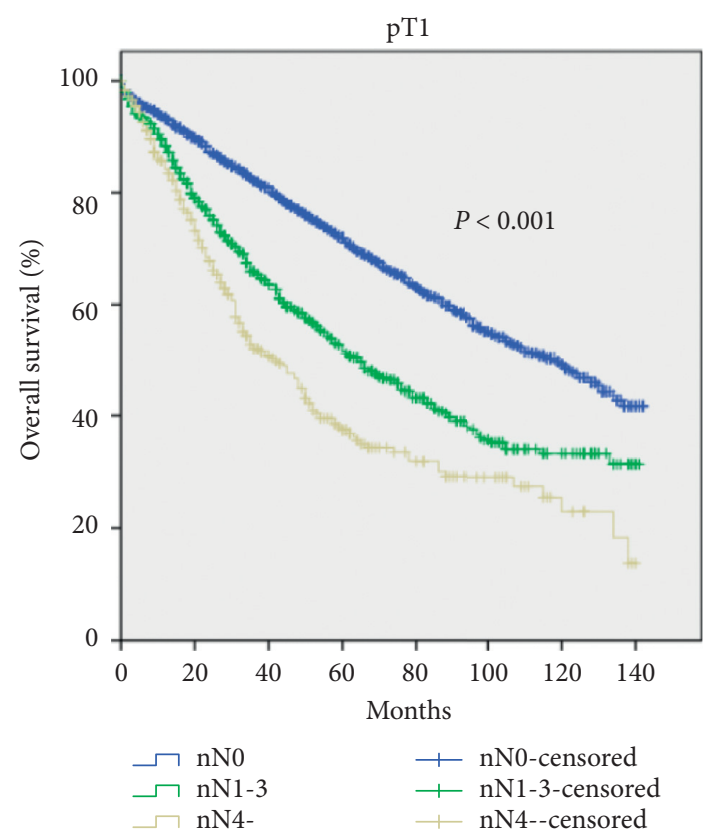

(a)

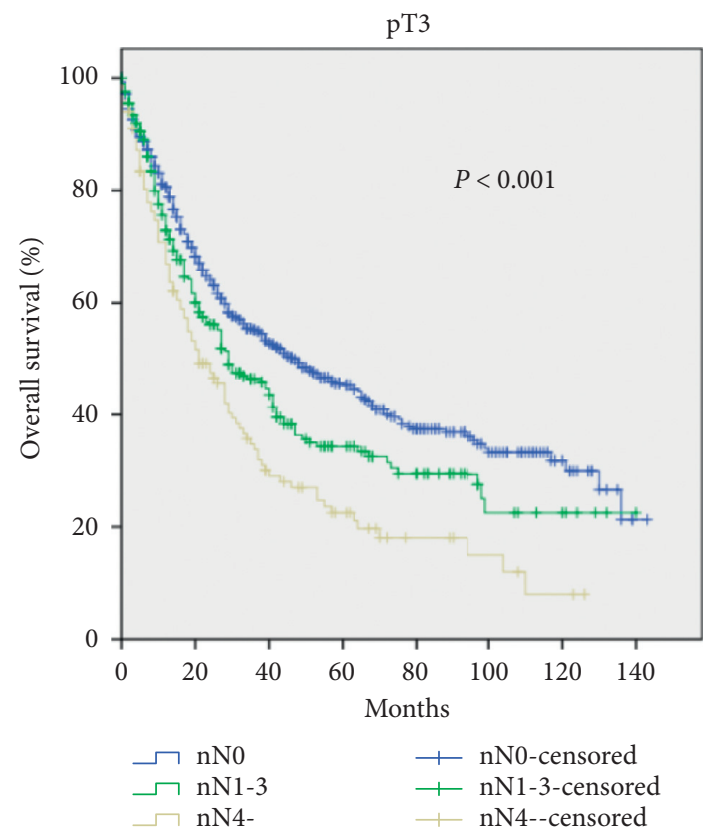

(c)

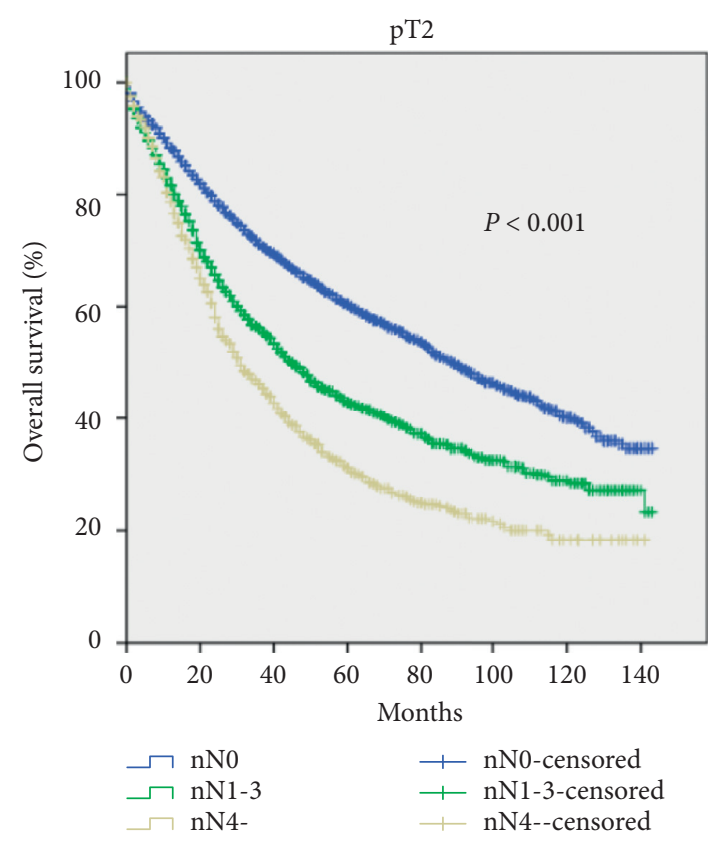

(b)

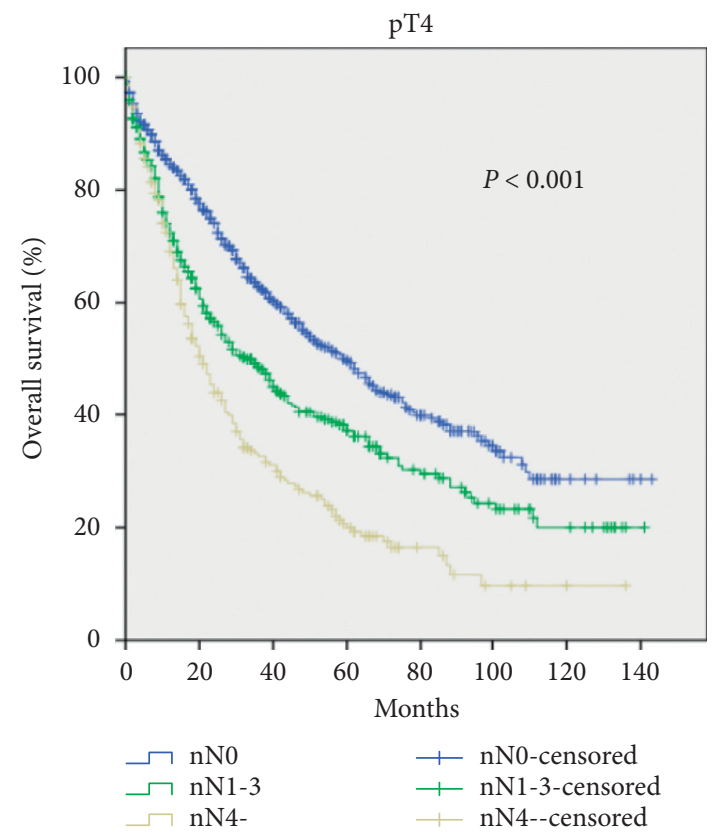

(d)

FIgure 3: Survival curves of different $\mathrm{nN}$ stages based on different $\mathrm{T}$ stages. Survival curves of different $\mathrm{nN}$ stages based on (a) pT1 stage $(P<0.001)$, (b) pT2 stage $(P<0.001)$, (c) pT3 stage $(P<0.001)$, and $(\mathrm{d})$ pT4 stage $(P<0.001)$.

point in this study was as follows: proper ways to accurately classify the number of metastatic LNs. Among the previous studies, there were some subgroups of $\mathrm{nN}$ classification, but they were just divided randomly or empirically. In our study, we reasonably classified the number of metastatic LNs into three subgroups using the X-tile model. It accurately determined the category of $\mathrm{nN}$, which made the further study more convincing.

The $\mathrm{nN}$ stage was well agreed with the $\mathrm{pN}$ stage, which were both favorable prognostic factors of OS. However, the definition of the $\mathrm{pN}$ stage was too extensive and inaccurately sufficient to evaluate the tumor progression. Thus, the number of metastatic LNs might provide significant information to the existing TNM staging system and could be a complement to the $\mathrm{pN}$ stage. Moreover, the survival curves of hypothetical $\mathrm{nN}$ stage in Figure 2(b) have also been confirmed in our previous study [21]. Although there were significant differences in the enrolled patients, the survival curves of $\mathrm{nN}$ stage were similar, suggesting that it was feasible to incorporate the number of lymph nodes into the 
TABLE 2: Influence of different variables on overall survival (OS) by pN stage for patients with NSCLC analyzed by Cox proportional hazard model.

\begin{tabular}{|c|c|c|c|c|}
\hline \multirow{2}{*}{ Variables } & \multicolumn{2}{|c|}{ Univariate analysis } & \multicolumn{2}{|c|}{ Multivariate analysis } \\
\hline & Wald $\chi^{2}$ & $P$ & HR $(95 \%$ CI $)$ & $P$ \\
\hline Age & 286.45 & $<0.001$ & & $<0.001$ \\
\hline$<65$ & & & Reference & \\
\hline$\geq 65$ & & & $1.578(1.497-1.664)$ & $<0.001$ \\
\hline Race & 18.08 & $<0.001$ & & $<0.001$ \\
\hline White & & & Reference & \\
\hline Black & & & $1.019(0.927-1.121)$ & 0.691 \\
\hline Others & & & $0.799(0.719-0.887)$ & $<0.001$ \\
\hline Sex & 134.46 & $<0.001$ & & $<0.001$ \\
\hline Female & & & Reference & \\
\hline Male & & & $1.359(1.290-1.431)$ & $<0.001$ \\
\hline Histology & 48.72 & $<0.001$ & & $<0.001$ \\
\hline Squamous & & & Reference & \\
\hline Adenocarcinoma & & & $0.831(0.789-0.875)$ & $<0.001$ \\
\hline pT stage & 273.10 & $<0.001$ & & $<0.001$ \\
\hline pT1 & & & Reference & \\
\hline pT2 & & & $1.345(1.267-1.429)$ & $<0.001$ \\
\hline pT3 & & & $1.951(1.770-2.151)$ & $<0.001$ \\
\hline pT4 & & & $1.837(1.679-2.011)$ & $<0.001$ \\
\hline pN stage & 633.92 & $<0.001$ & & $<0.001$ \\
\hline pNo & & & Reference & \\
\hline pN1 & & & $1.630(1.534-1.733)$ & $<0.001$ \\
\hline $\mathrm{pN} 2$ & & & $2.157(2.023-2.300)$ & $<0.001$ \\
\hline $\mathrm{pN} 3$ & & & $2.799(1.983-3.949)$ & $<0.001$ \\
\hline
\end{tabular}

TABLE 3: Influence of different variables on overall survival (OS) for patients by $\mathrm{nN}$ stage with NSCLC analyzed by Cox proportional hazard model.

\begin{tabular}{|c|c|c|c|c|}
\hline \multirow{2}{*}{ Variables } & \multicolumn{2}{|c|}{ Univariate analysis } & \multicolumn{2}{|c|}{ Multivariate analysis } \\
\hline & Wald $\chi^{2}$ & $P$ & HR (95\% CI) & $P$ \\
\hline Age & 269.89 & $<0.001$ & & $<0.001$ \\
\hline$<65$ & & & Reference & \\
\hline$\geq 65$ & & & $1.555(1.475-1.639)$ & $<0.001$ \\
\hline Race & 17.84 & $<0.001$ & & $<0.001$ \\
\hline White & & & Reference & \\
\hline Black & & & $1.022(0.930-1.124)$ & 0.649 \\
\hline Others & & & $0.801(0.721-0.889)$ & $<0.001$ \\
\hline Sex & 137.28 & $<0.001$ & & $<0.001$ \\
\hline Female & & & Reference & \\
\hline Male & & & $1.363(1.294-1.435)$ & $<0.001$ \\
\hline Histology & 49.25 & $<0.001$ & & $<0.001$ \\
\hline Squamous & & & Reference & \\
\hline Adenocarcinoma & & & $0.831(0.789-0.875)$ & $<0.001$ \\
\hline pT stage & 286.01 & $<0.001$ & & $<0.001$ \\
\hline $\mathrm{pT} 1$ & & & Reference & \\
\hline pT2 & & & $1.340(1.261-1.422)$ & $<0.001$ \\
\hline pT3 & & & $1.967(1.785-2.169)$ & $<0.001$ \\
\hline pT4 & & & $1.870(1.709-2.046)$ & $<0.001$ \\
\hline $\mathrm{nN}$ stage & 696.79 & $<0.001$ & & $<0.001$ \\
\hline nNo & & & Reference & \\
\hline $\mathrm{nN} 1-3$ & & & $1.657(1.564-1.756)$ & $<0.001$ \\
\hline nN4- & & & $2.371(2.213-2.540)$ & $<0.001$ \\
\hline
\end{tabular}

current TNM stage. Since the primary tumor's characteristic was an essential factor to determine treatment strategy and prognostic care, we also compared the OS in each pT stage based on the $\mathrm{nN}$ classification. As expected, whatever the $\mathrm{pT}$ category was, $\mathrm{nN}$ classification exhibited an excellent prognostic predictive value. It indicated that $\mathrm{nN}$ was an excellent parameter that could be considered as a classification factor. 


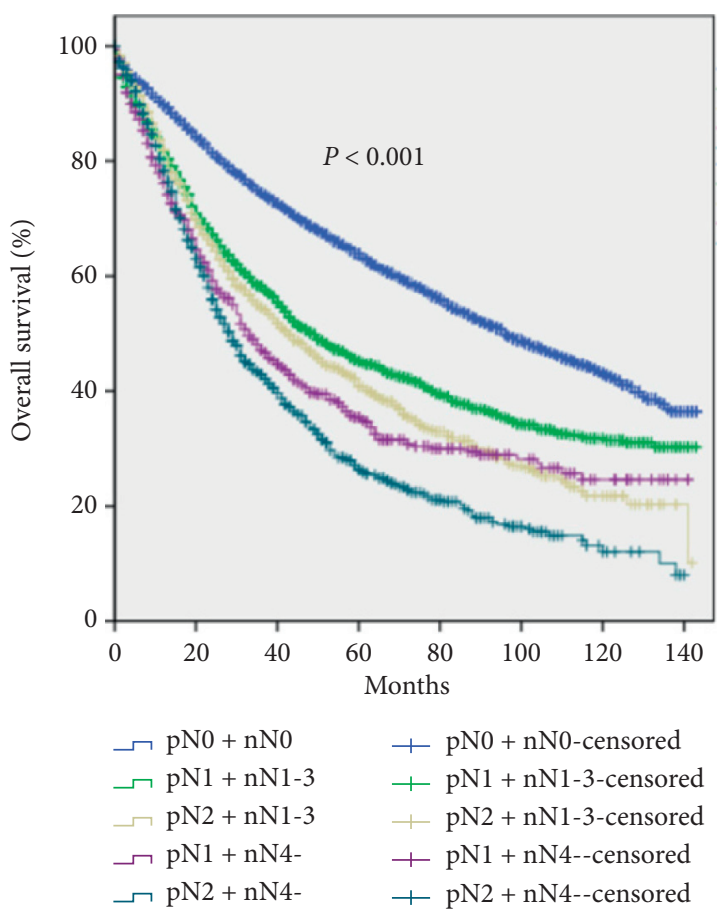

Figure 4: Survival curves of patients with stages $\mathrm{pN} 0+\mathrm{nN} 0$, $\mathrm{pN} 1+\mathrm{nN} 1-3, \quad \mathrm{pN} 2+\mathrm{nN} 1-3, \quad \mathrm{pN} 1+\mathrm{nN} 4-$, and $\mathrm{pN} 2+\mathrm{nN} 4-$, respectively $(P<0.001)$.

Thus, we provided a novel classification that combined $\mathrm{pN}$ stage with the $\mathrm{nN}$ stage for nodal involvement assessment. In conclusion, the subcategory of the OS was significantly distinguished. When we divided $\mathrm{pN} 1$ into two subgroups by $\mathrm{nN}$, the OS decreased with higher PLN number. Furthermore, a similar observation was found with pN2 stage and the subgroups. One group [14] also performed similar analysis, but no exact conclusion was demonstrated. They did not show a significant difference in $\mathrm{pN} 0$ and $\mathrm{pN} 1+\mathrm{nN} 1-3$ stages in their studies, but in this study, a significant difference was observed. Survival differences were confusing on the two nN4- groups. However, when we divided nN4- into two subgroups, $\mathrm{pN} 1+\mathrm{nN} 4$ - and $\mathrm{pN} 2+\mathrm{nN} 4-$, the survival clearly decreased with more anatomical metastasis. Our study has provided substantial evidence that the new classification had better prognostic indication than the old TNM staging system.

Interestingly, the survival was higher in $\mathrm{pN} 2+\mathrm{nN} 1-3$ compared to that of $\mathrm{pN} 1+\mathrm{nN} 4$ - within the first 5 years. It indicated that the location of metastatic LN played a prominent role for the prognosis in the early time. Hence, proper postoperative treatment and care could be better applied with different groups to have a longer survival time. Some studies showed that both the location and disease burden could affect the prognosis, but the differences were unknown. In this study, we demonstrated that a combination of $\mathrm{pN}$ and $\mathrm{nN}$ significantly distinguished the classification, particularly the cutting edge of $\mathrm{pN} 1$ and $\mathrm{pN} 2$.

However, there were still some limitations when considering $\mathrm{nN}$ stage as a classification criterion. It is more like a pathologically proof for prognosis rather than a determinant factor for proper treatment. Thus, an accurate method that could identify all the PLNs is significantly required. Although various methods could be used to evaluate the malignancy, such as magnetic resonance imaging, positron emission tomography scan, fine needle aspiration biopsy, and specific tumor markers, none of them were sufficiently reliable. Hence, with the advancement of modern technology, better methods are significantly required. Since it is difficult to include the number of all PLNs, we need to combine the $\mathrm{pN}$ stage with the $\mathrm{nN}$ stage to evaluate the OS. Data from the SEER database are mainly based on White patients; thus, investigation regarding cancer staging system on other racial groups is also significantly important. Therefore, further study is required to evaluate if $\mathrm{nN}$ should be involved in the TNM staging system.

\section{Conclusion}

In our study, we found that the $\mathrm{nN}$ stage was a significant prognostic factor on OS for NSCLC patients. Furthermore, the combination of the anatomical location and the number of LNs involvement in NSCLC patients had a better prognostic value than the current TNM staging system based on only $\mathrm{pN}$ stage. These results also need further studies to confirm the validity of the novel TNM staging system.

\section{Abbreviations}

NSCLC: Non-small cell lung cancer

TNM: Tumor-node-metastasis

LNs: Lymph nodes

PLNs: Positive lymph nodes

SEER: Surveillance epidemiology and end results

HR: Hazard ratio

CI: Confidence interval

OS: Overall survival

pN: Pathological LNs

$\mathrm{nN}$ : Number of LNs

CSS: Cancer-specific survival

NLNS: The number of lymph nodes sampled.

\section{Data Availability}

The datasets used and analyzed during the current study are available from the corresponding author on reasonable request.

\section{Conflicts of Interest}

All the authors have no conflicts of interest to declare.

\section{Authors' Contributions}

Xiaoling Shang contributed equally to this work.

\section{References}

[1] R. L. Siegel, K. D. Miller, and A. Jemal, "Cancer statistics, 2017," CA: A Cancer Journal for Clinicians, vol. 67, no. 1, pp. 7-30, 2017. 
[2] C. Le Péchoux, "Role of postoperative radiotherapy in resected non-small cell lung cancer: a reassessment based on new data," Oncologist, vol. 16, no. 5, pp. 672-681, 2011.

[3] P. Goldstraw, K. Chansky, J. Crowley et al., "The IASLC lung cancer staging project: proposals for revision of the TNM stage groupings in the forthcoming (eighth) edition of the TNM classification for lung cancer," Journal of Thoracic Oncology, vol. 11, no. 1, pp. 39-51, 2016.

[4] P. Goldstraw, J. Crowley, K. Chansky et al., "The IASLC lung cancer staging project: proposals for the revision of the TNM stage groupings in the forthcoming (seventh) edition of the TNM classification of malignant tumours," Journal of Thoracic Oncology, vol. 2, no. 8, pp. 706-714, 2009.

[5] W. E. Eberhardt, A. Mitchell, J. Crowley et al., "The IASLC lung cancer staging project: proposals for the revision of the $M$ descriptors in the forthcoming eighth edition of the TNM classification of lung cancer," Journal of Thoracic Oncology, vol. 10, no. 11, pp. 1515-1522, 2016.

[6] L. H. Sobin and M. K. W. C. Gospodarowicz, TNM Classification of Malignant Tumors, Wiley-Blackwell, Oxford, UK, 7th edition, 2010.

[7] R. Rami-porta, V. Bolejack, D. J. Giroux et al., "The IASLC lung cancer staging project: the new database to inform the eighth edition of the TNM classification of lung cancer," Journal of Thoracic Oncology, vol. 9, no. 11, pp. 1618-1624, 2014.

[8] R. J. Cerfolio, B. Ojha, A. S. Bryant, V. Raghuveer, J. M. Mountz, and A. A. Bartolucci, "The accuracy of integrated PET-CT compared with dedicated pet alone for the staging of patients with nonsmall cell lung cancer," The Annals of Thoracic Surgery, vol. 78, no. 3, pp. 1017-1023, 2004.

[9] J. E. Tepper, M. J. O 'connell, D. Niedzwiecki et al., "Impact of number of nodes retrieved on outcome in patients with rectal cancer," Journal of Clinical Oncology, vol. 19, no. 1, pp. 157-163, 2016.

[10] L. Weir, C. Speers, Y. D'yachkova, and I. A. Olivotto, "Prognostic significance of the number of axillary lymph nodes removed in patients with node-negative breast cancer," Journal of Clinical Oncology, vol. 20, no. 7, pp. 1793-1799, 2002.

[11] S. Wei, H. Asamura, R. Kawachi, H. Sakurai, and S.-i. Watanabe, "Which is the better prognostic factor for resected non-small cell lung cancer: the number of metastatic lymph nodes or the currently used nodal stage classification?" Journal of Thoracic Oncology, vol. 6, no. 2, pp. 310-318, 2011.

[12] H. W. Herr, B. H. Bochner, G. Dalbagni, S. M. Donat, V. E. Reuter, and D. F. Bajorin, "Impact of the number of lymph nodes retrieved on outcome in patients with muscle invasive bladder cancer," Journal of Urology, vol. 167, no. 3, pp. 1295-1298, 2002.

[13] H. Saji, M. Tsuboi, K. Yoshida et al., "Prognostic impact of number of resected and involved lymph nodes at complete resection on survival in non-small cell lung cancer," Journal of Thoracic Oncology, vol. 6, no. 11, pp. 1865-1871, 2011.

[14] H. Saji, M. Tsuboi, Y. Shimada et al., "A proposal for combination of total number and anatomical location of involved lymph nodes for nodal classification in non-small cell lung cancer," Chest, vol. 143, no. 6, pp. 1618-1625, 2013.

[15] H. Asamura, K. Chansky, J. Crowley et al., "The international association for the study of lung cancer lung cancer staging project: proposals for the revision of the $\mathrm{N}$ descriptors in the forthcoming 8th edition of the TNM classification for lung cancer," Journal of Thoracic Oncology, vol. 10, no. 12, pp. 1675-1684, 2015.
[16] J. G. Lee, C. Y. Lee, I. K. Park et al., "Number of metastatic lymph nodes in resected non-small cell lung cancer predicts patient survival," The Annals of Thoracic Surgery, vol. 85, no. 1, pp. 211-215, 2008.

[17] T. Fukui, S. Mori, K. Yokoi, and T. Mitsudomi, "Significance of the number of positive lymph nodes in resected non-small cell lung cancer," Journal of Thoracic Oncology, vol. 1, no. 2, pp. 120-125, 2006.

[18] E. A. David, D. T. Cooke, Y. Chen, K. Nijar, R. J. Canter, and R. D. Cress, "Does lymph node count influence survival in surgically resected non-small cell lung cancer?” The Annals of Thoracic Surgery, vol. 103, no. 1, pp. 226-235, 2017.

[19] J. M. Varlotto, A. Recht, M. Nikolov, J. C. Flickinger, and M. M. DeCamp, "Extent of lymphadenectomy and outcome for patients with stage I nonsmall cell lung cancer," Cancer, vol. 115 , no. 4 , pp. 851-858, 2009.

[20] M. S. Ludwig, M. Goodman, D. L. Miller, and P. A. S. Johnstone, "Postoperative survival and the number of lymph nodes sampled during resection of node-negative nonsmall cell lung cancer," Chest, vol. 128, no. 3, pp. 1545-1550, 2005.

[21] Y. Fan, Y. Du, W. Sun, and H. Wang, "Including positive lymph node count in the AJCC N staging may be a better predictor of the prognosis of NSCLC patients, especially stage III patients: a large population-based study," International Journal of Clinical Oncology, vol. 24, no. 11, pp. 1359-1366, 2019. 